\title{
Pharmacokinetics of moxifloxacin and linezolid during and after pregnancy in a patient with multidrug-resistant tuberculosis
}

\author{
To the Editor:
}

Worldwide, tuberculosis (TB) is among the top five causes of death for women aged 15-44 years [1]. In 2014, an estimated 480000 of newly reported TB cases were multidrug-resistant (MDR) TB [1]. Pregnancy is a risk factor for reactivation of TB, but data about multidrug-resistant (MDR)-TB in pregnant women are lacking. There are few data about the efficacy and safety of second-line anti-TB drugs during pregnancy for both the mother and the unborn child [2]. One study from Peru showed a mortality rate of $13 \%$ in 38 patients and $13 \%$ of these 38 patients were lost to follow-up. Five of the pregnancies ended in spontaneous abortions, and one child was stillborn. Data for pregnant women did not differ from the general MDR-TB population in Peru [2].

To assure efficacy while minimising adverse events, therapeutic drug monitoring (TDM) has been proposed [3]. In TDM of anti-TB drugs, the accurately measured exposure to the drug [4] is evaluated in relation to the minimum inhibitory concentration (MIC) of the offending strain of Mycobacterium tuberculosis [5]. Low drug exposure is associated with an increased risk of treatment failure or with acquired resistance. High drug exposure might result in increased toxicity and adverse events [6]. Little is known about the effects of pregnancy in patients with MDR-TB with regards to the pharmacokinetics of second-line anti-TB drugs [2]. During pregnancy, pharmacokinetic parameters might change over time because of dynamic physiological changes in different stages of pregnancy, leading to inadequate treatment and poor outcome. In this case study, we aimed to describe the pharmacokinetics of moxifloxacin (Mfx) and linezolid (Lzd) during and after pregnancy in a patient with MDR-TB.

A 25-year-old HIV-negative female from Somalia presented with a cough of several months' duration. She had arrived in the Netherlands 10 months earlier [7]. Her sister had been diagnosed with TB 12 years ago, and had been treated successfully. Physical examination revealed an enlarged lymph node in her neck. Culture from a fine-needle aspirate yielded $M$. tuberculosis. The chest radiograph showed no abnormalities; she was started on rifampin, isoniazid, pyrazinamide and ethambutol. Molecular susceptibility testing of the cultured isolate showed mutations in both the rpoB and katG genes. She was diagnosed with MDR-TB and transferred to our TB reference centre at 11 weeks' gestation. The first-line anti-TB drugs were discontinued and we decided to wait for further susceptibility results before starting with-second line anti-TB drugs to avoid giving inactive but potentially toxic drugs to the fetus. We considered it safe to withhold the treatment for some time as lymph node TB is paucibacillary and she was clinically stable without apparent disease activity apart from the nuchal lymph nodes. In vitro phenotypical susceptibility testing was performed, and the isolate appeared resistant to the first-line drugs rifampicin, isoniazid and ethambutol, but susceptible to all injectables, moxifloxacin, prothionamide, linezolid, clofazimine and co-trimoxazole. Treatment was started at week 20 of gestation. She was treated with pyrazinamide (1 $750 \mathrm{mg}$ once daily), Mfx (400 mg once daily), prothionamide (250 mg in the morning and $500 \mathrm{mg}$ in the evening) and Lzd (300 mg twice daily). 1 month later, the laboratory reported pyrazinamide resistance and this drug was subsequently stopped. Because of persistent vomiting, prothionamide was replaced by clofazimine after 1 month.

@ERSpublications

Pharmacokinetics of moxifloxacin and linezolid during and after pregnancy in an MDR-TB patient http://ow.ly/Ind2308nLlB

Cite this article as: Van Kampenhout E, Bolhuis MS, Alffenaar J-WC, et al. Pharmacokinetics of moxifloxacin and linezolid during and after pregnancy in a patient with multidrug-resistant tuberculosis. Eur Respir J 2017; 49: 1601724 [https://doi.org/10.1183/13993003.01724-2016]. 
TDM is the standard care in our centre for patients with MDR-TB [8]. We hypothesised that her pregnancy would cause changes in her volume of distribution; we therefore performed TDM at three different time-points, i.e. a couple of weeks after start of treatment $(25+5$ weeks of pregnancy), at 1 month before delivery ( $35+5$ weeks of pregnancy) and when the recovery of the mother had normalised (18 weeks post partum).

The following areas under the concentration-time curves (AUCs) were measured (figure 1). At 25+5 weeks, the $\mathrm{AUC}_{0-24 \mathrm{~h}}$ for $\mathrm{Lzd} 300 \mathrm{mg}$ twice a day and $\mathrm{Mfx} 400 \mathrm{mg}$ once a day were $48 \mathrm{mg} \cdot \mathrm{h}^{-1} \cdot \mathrm{L}^{-1}$ and $31.6 \mathrm{mg} \cdot \mathrm{h}^{-1} \cdot \mathrm{L}^{-1}$, respectively. At $35+5$ weeks, the Lzd $\mathrm{AUC}_{0-24 \mathrm{~h}}$ was $106 \mathrm{mg} \cdot \mathrm{h}^{-1} \cdot \mathrm{L}^{-1}$ and the Mfx $\mathrm{AUC}_{0-24 \mathrm{~h}}$ was $32 \mathrm{mg} \cdot \mathrm{h}^{-1} \cdot \mathrm{L}^{-1}$. Approximately 18 weeks post partum, the $\mathrm{Lzd}$ and $\mathrm{Mfx} \mathrm{AUC}_{0-24 \mathrm{~h}}$ were 203 and $34.9 \mathrm{mg} \cdot \mathrm{h}^{-1} \cdot \mathrm{L}^{-1}$, respectively.

MICs were determined to be $0.5 \mathrm{mg} \cdot \mathrm{L}^{-1}$ for $\mathrm{Lzd}$ and $0.125 \mathrm{mg} \cdot \mathrm{L}^{-1}$ for $\mathrm{Mfx}$ by the Dutch National Mycobacterial Reference Laboratory (Bilthoven, the Netherlands) using the Middlebrook 7H10 agar dilution method. As the AUCs of linezolid and moxifloxacin were adequate over time, no dose adjustment of either drug was performed.

5 months post partum (results of the last result were not known yet), her linezolid was discontinued due to polyneuropathy. She continued with cotrimoxazole for the rest of her treatment [9]. The moxifloxacin and clofazimine were continued for the rest of the treatment, in total 20 months. At the time of writing, the patient was well and her child appeared healthy.

In our patient, we observed a decreased exposure of Lzd and Mfx during pregnancy compared with post partum measurements. We saw a trend towards an increased exposure of Lzd and Mfx from $25+5$ to $35+5$ weeks of pregnancy.

Because we obtained the third pharmacokinetic blood curve 18 weeks after pregnancy, we hypothesised that physiological changes occurring during pregnancy would be reversed and we considered these samples to be representative for a non-pregnant patient. Mfx exposure was similar to a previous study conducted in our centre $(n=16)$ where patients had a median (interquartile range (IQR)) exposure of 24.8 $(20.7-35.2) \mathrm{mg} \cdot \mathrm{h}^{-1} \cdot \mathrm{L}^{-1}$ [10]. Lzd exposure after pregnancy was higher than observed in a previous study in patients receiving a similar dose of $300 \mathrm{mg}$ twice daily, i.e. median (IQR) exposure of 72.6 (66.492.6) $\mathrm{mg} \cdot \mathrm{h}^{-1} \cdot \mathrm{L}^{-1}[11]$.

There are several different possible explanations for the lower AUC during pregnancy. The volume of body fluid may increase by about 6-8 L with plasma volume expansion of $50 \%$. As a consequence, the volume of distribution of drugs, in particular hydrophilic drugs with a relatively small volume of distribution, may increase resulting in a decreased peak serum concentration [12]. Elimination of medication may be enhanced during pregnancy. Hepatic blood flow increases and there may be a higher rate of hepatic metabolism of some drugs [12]. The metabolic activity of enzymes, such as CYP3A4, increases during pregnancy [12]. Lzd is believed to be a substrate for CYP3A4 [13]. Furthermore, in pregnant women renal plasma flow increases by $25-50 \%$ and glomerular filtration rate by $50 \%$, resulting in faster elimination. Physiological and pharmacokinetic changes are dynamic throughout gestation and dosage adjustments may be necessary during each trimester [12]. Nausea and vomiting potentially reduce drug absorption
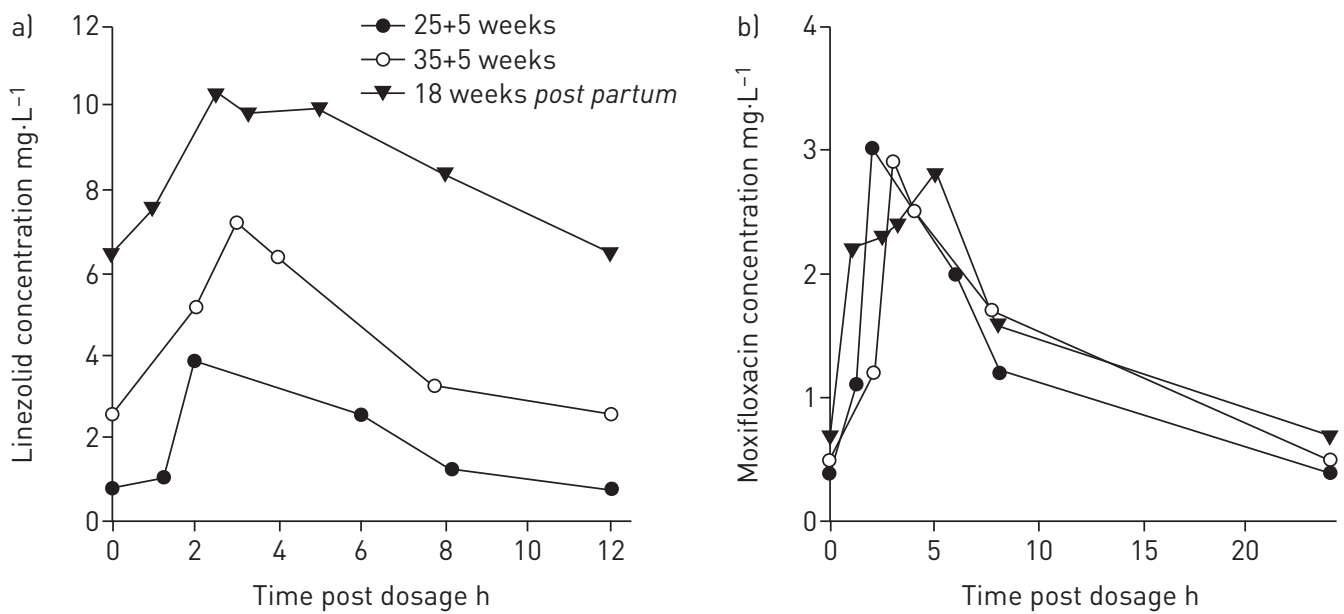

FIGURE 1 a) Linezolid and b) moxifloxacin concentration-time curves at $25+5$ weeks weighing $75.5 \mathrm{~kg}$ (solid circles); $35+5$ weeks weighing $74 \mathrm{~kg}$ (open circles); and 18 weeks post partum weighing $68 \mathrm{~kg}$ (solid triangles) 
during the first trimester of pregnancy. During pregnancy, gastric emptying and intestinal motility are reduced [14]. Body weight increases during pregnancy, and hormonal changes may influence drug concentrations and increased hepatic blood flow may stimulate hepatic metabolism of some drugs [14].

To our best knowledge, this is the first study describing TDM in such a challenging situation. Here, we only report a single case study; another limitation is the long period between childbirth and PK samples taken after childbirth. The polyneuropathy experienced might have been prevented if the dose of linezolid had been lowered at an earlier stage. In retrospect, we should perhaps have obtained PK samples 2-4 weeks post partum.

As proper drug choice is even more important in women with TB and pregnancy, we need to know more about the pharmacokinetics of second-line TB-medication in pregnancy. All pregnant patients with MDR-TB should have TDM during all trimesters, shortly post partum and several months later if needed. Dried blood spots and limited sampling strategies may help to facilitate these ambitions [15].

Els Van Kampenhout ${ }^{1,6}$, Mathieu S. Bolhuis ${ }^{2,6}$, Jan-Willem C. Alffenaar ${ }^{2}$, Laurien M.A. Oswald ${ }^{3}$, Huib A.M. Kerstjens ${ }^{1}$, Wiel C.M. de Lange ${ }^{1,4}$, Tjip S. van der Werf ${ }^{1,5}$ and Onno W. Akkerman ${ }^{1,4}$

${ }^{1}$ University of Groningen, University Medical Center Groningen, Dept of Pulmonary Diseases and Tuberculosis, Groningen, The Netherlands. ${ }^{2}$ University of Groningen, University Medical Center Groningen, Dept of Clinical Pharmacy and Pharmacology, Groningen, The Netherlands. ${ }^{3}$ Franciscus Gasthuis en Vlietland Teaching Hospital, Rotterdam, The Netherlands. ${ }^{4}$ University of Groningen, University Medical Center Groningen, Tuberculosis Center Beatrixoord, Haren, The Netherlands. ${ }^{5}$ University of Groningen, University Medical Center Groningen, Dept of Internal Medicine, Groningen, The Netherlands. ${ }^{6}$ Both authors contributed equally.

Correspondence: O.W. Akkerman, University Medical Center Groningen, Tuberculosis Center Beatrixoord, Dilgtweg 5, 9751 ND Haren, Groningen, The Netherlands. E-mail: o.w.akkerman@umcg.nl

Received: Aug 302016 | Accepted after revision: Nov 252016

Conflict of interest: Disclosures can be found alongside this article at erj.ersjournals.com

\section{References}

1 WHO: Global Tuberculosis Report 2015. Geneva, World Health Organization, 2015. Available at: www.who.int/tb/ publications/global_report/en/

2 Palacios E, Dallman R, Muñoz M, et al. Drug-resistant tuberculosis and pregnancy: treatment outcomes of 38 cases in Lima, Peru. Clin Infect Dis 2009; 48: 1413-1419.

3 Zuur MA, Bolhuis MS, Anthony R, et al. Current status and opportunities for therapeutic drug monitoring in the treatment of tuberculosis. Expert Opin Drug Metab Toxicol 2016; 12: 509-521.

4 Aarnoutse RE, Sturkenboom MGG, Robijns $\mathrm{K}$, et al. An interlaboratory quality control programme for the measurement of tuberculosis drugs. Eur Respir J 2015; 46: 268-271.

5 Srivastava S, Peloquin CA, Sotgiu G, et al. Therapeutic drug management: is it the future of multidrug-resistant tuberculosis treatment? Eur Respir J 2013; 42: 1449-1453.

6 De Lorenzo S, Alffenaar JW, Sotgiu G, et al. Efficacy and safety of meropenem-clavulanate added to linezolid-containing regimens in the treatment of MDR-/XDR-TB. Eur Respir J 2013; 41: 1386-1392.

7 de Vries G, van Rest J, Meijer W, et al. Preventing and controlling tuberculosis among refugees in Europe: more needed for high-risk populations. Eur Respir J 2016; 48: 274-276.

8 Bolhuis MS, Tiberi S, Sotgiu G, et al. Is there still room for therapeutic drug monitoring of linezolid in patients with tuberculosis? Eur Respir J 2016; 47: 1288-1290.

9 Alsaad N, Wilffert B, van Altena R, et al. Potential antimicrobial agents for the treatment of multidrug-resistant tuberculosis. Eur Respir J 2014; 43: 884-897.

10 Pranger AD, van Altena R, Aarnoutse RE, et al. Evaluation of moxifloxacin for the treatment of tuberculosis: 3 years of experience. Eur Respir J 2011; 38: 888-894.

11 Bolhuis MS, van Altena R, van Soolingen D, et al. Clarithromycin increases linezolid exposure in multidrug-resistant tuberculosis patients. Eur Respir J 2013; 42: 1614-1621.

12 Matsui DM. Therapeutic drug monitoring in pregnancy. Ther Drug Monit 2012; 34: 507-511.

13 Gandelman K, Zhu T, Fahmi OA, et al. Unexpected effect of rifampin on the pharmacokinetics of linezolid: in silico and in vitro approaches to explain its mechanism. J Clin Pharmacol 2011; 51: 229-236.

14 Dawes M, Chowienczyk PJ. Drugs in pregnancy. Pharmacokinetics in pregnancy. Best Pract Res Clin Obstet Gynaecol 2001; 15: 819-826.

15 Ghimire S, Bolhuis MS, Sturkenboom MGG, et al. Incorporating therapeutic drug monitoring into the World Health Organization hierarchy of tuberculosis diagnostics. Eur Respir J 2016; 47: 1867-1869. 